\title{
Klassiker als Zugang zur Wissenschaft: Eine Einladung zur Beteiligung
}

\author{
Peter Tremp
}

\section{Zusammenfassung}

Die Studieneingangsphase kann als Einstieg in die wissenschaftliche Welt der Hochschulen gesehen werden, Studierende sind hier mit den spezifischen Denkweisen einer Disziplin konfrontiert. Wie kann die Wissenschaftssozialisation didaktisch bearbeitet werden, welche methodischen Zugänge haben sich etabliert? Und: Welche Bedeutung haben Klassiker einer Disziplin für den Aufbau einer wissenschaftlich-disziplinären Identität? Der Beitrag erörtert solche Fragen und diskutiert, inwiefern diese Überlegungen auch für die Disziplin resp. das Arbeitsfeld Hochschuldidaktik gelten.

\section{Schlüsselwörter}

Wissenschaftssozialisation · Studieneingangsphase · Diskursivität

Hochschulen sind wissenschaftliche Einrichtungen. In ihrer Forschungstätigkeit generieren sie neues wissenschaftliches Wissen, überprüfen die Reichweite bestehenden Wissens und entwickeln Methoden der wissenschaftlichen Erkenntnisgewinnung weiter. Und mit ihren Studienangeboten leisten Hochschulen einen bedeutsamen Beitrag zur wissenschaftlichen Sozialisation und Qualifizierung des Nachwuchses für akademische Berufsfelder - und gleichzeitig zur Weiterentwicklung der Disziplinen und zur eigenen Reproduktion.

P. Tremp $(\bowtie)$

Zentrum für Hochschuldidaktik,

Pädagogische Hochschule Luzern, Luzern, Schweiz

E-Mail: peter.tremp@phlu.ch

(C) Springer Fachmedien Wiesbaden GmbH, ein Teil von Springer Nature 2020 
Ein Hochschulstudium ist entsprechend als Einstieg in die wissenschaftliche Welt konzipiert. In dieser programmatisch betonten Wissenschaftsorientierung und damit verknüpft durch die thematische Fokussierung des Studiums unterscheiden sich Hochschulen von den vorangehenden Stufen des Bildungssystems. Der Übergang ins Studium und also in diese Wissenschaftswelt bietet einige Herausforderungen. Insbesondere stellt sich die Frage, wie es gelingt, eine wissenschaftlich-disziplinäre Identität aufzubauen, wie es gelingt, in die Denkweise einer Disziplin hineinzufinden.

Damit sind Fragen nach didaktischen Zugängen zum Zwecke der Wissenschaftssozialisation (insbesondere in der Studieneingangsphase) verbunden und insbesondere nach der Bedeutung von Klassikern. Im vorliegenden Beitrag werden in einem ersten Kapitel ausgewählte Zugänge beschrieben, welche diese Funktion der Wissenschaftssozialisation betonen. Im zweiten Kapitel wird die didaktische Bedeutung von Klassikern diskutiert, bevor in einem abschließenden dritten Kapitel gefragt wird, inwiefern diese Überlegungen auch auf die Disziplin resp. das Arbeitsfeld Hochschuldidaktik übertragen werden können.

\section{Didaktische Zugänge zur Wissenschaftssozialisation}

Die Didaktik ist die Lehre der erfolgsversprechenden Zugänge zu einer sich anzueignenden Sache. Insofern hat die Didaktik eine Reihe von Vorschlägen entwickelt, wie Lernende denn tatsächlich mit dieser Sache konfrontiert werden. Vor allem wird die Bedeutung des Beginns betont: Wie kann die Begegnung mit der Sache gestartet, wie ein Anfang gemacht werden?

Während sich in der hochschuldidaktischen Diskussion diese Überlegungen insbesondere auf eine einzelne Vorlesungssequenz oder einen Seminartermin, gelegentlich auch auf eine gesamte Veranstaltungsreihe bezogen haben, wurde in den letzten Jahren vermehrt die Bedeutung der Studieneingangsphase diskutiert und damit die Frage gestellt, wie die zumeist disziplinär organisierte Wissenschaftssozialisation starten kann. Wie finden sich Studienanfängerinnen und -anfänger in der Wissenschaftswelt ein, wie sie in Hochschulen repräsentiert wird? Wie gelingt es, eine wissenschaftlich-disziplinäre Identität aufzubauen und in die Denkweise einer Disziplin hineinzufinden mit ihrem spezifischen Wissenskorpus sowie hinsichtlich der Methoden und Zugänge der Disziplin (Eugster und Tremp 2018)? Wie wird die Besonderheit des wissenschaftlichen Wissens und damit auch der Hochschulen erfahrbar, also das, was Humboldt als „Eigentümlichkeit der höheren wissenschaftlichen Anstalten“ festgehalten hat, „dass sie die 
Wissenschaft immer als ein noch nicht ganz aufgelöstes Problem behandeln und daher immer im Forschen bleiben“ (Humboldt 1810/1964, S. 256)?

Eine besondere Bedeutung haben in diesem Zusammenhang einführende Lehrveranstaltungen oder Studienbücher, welche eine „Einführung in ...“ versprechen. Tatsächlich finden sich - beispielsweise bezogen auf die Disziplin Erziehungswissenschaft - eine Reihe von Publikationen, welche in ihren Hauptoder Untertiteln gerade dies versprechen.

Im Gegensatz zu Handbüchern sind diese Einführungen oftmals von einer Person (oder aber sehr wenigen Autorinnen und Autoren) verfasst, vor allem aber richten sie sich insbesondere an Novizinnen und Novizen der Disziplin, auch wenn die Systematik der Themendarstellung bisweilen an Handbücher erinnert.

Im Folgenden werden nun ausgewählte weitere Zugänge erörtert, die einen Einstieg in die Wissenschafts- und Hochschulwelt beabsichtigen und je mit spezifischen Zielsetzungen verbinden.

Als „Einführung in pädagogisches Sehen und Denken“ - so der Titel der Publikation - versteht sich eine Sammlung von Texten, welche 1967 erstmals von Andreas Flitner und Hans Scheuerl herausgegeben wurde und im Lehramtsstudium resp. im Studium der Erziehungswissenschaft lange eine große Bedeutung gehabt hat, inzwischen aber kaum mehr verwendet wird.

Wie wird diese Einführung nun realisiert, welcher Zugang wird gewählt? Ausgangspunkt einer pädagogischen Analyse und Erörterung bilden hier Alltagserscheinungen, Fälle und Phänomene. Die in diesem Buch versammelten Beispieltexte sind in Gruppen gegliedert, die ,in vielfachen Abwandlungen immer wieder auftauchen und eine ähnliche Struktur zeigen“ (Flitner und Scheuerl 1984, S. 239). Die Strukturierung ordnet sich in die drei Themen Erziehungsverhältnis zwischen Erwachsenem und Kind, soziale Ordnungen, die ,im Namen der Erziehungsverhältnisse geschaffen werden“ (ebd., S. 240) und schließlich „Denkund Erlebnisformen der Kinder" (ebd., S. 240). Mit dieser Zusammenstellung legen die beiden Herausgeber „einfache Fallbeschreibungen“ vor, „Fälle und Unfälle des Erziehungsalltags“, die das pädagogische Sehen lernen lassen und „auch das historische-systematische Denken auf die Probe stellen“ (ebd., S. 241).

Wie wird dies konkret realisiert? Der in diese Sammlung integrierte Text von Andreas Flitner („Unterstützung der Leistungsfähigkeit“) beginnt beispielsweise mit einer Situation an einem schulischen Elternabend und dem vehementen Einspruch eines Vaters auf die Erläuterung der Lehrerin zu ihrem Bewertungssystem (ebd., S. 153-158). Dieser „Fall“ ist Ausgangspunkt zu Überlegungen zum Begriff der Leistung. Unterschiedliche „Leistungen“ werden kurz erörtert, bevor dann eingehender die Schule als Leistungsraum eigener Art diskutiert wird. Damit bietet dieser Text nicht nur Gelegenheit, den Fall „Elternabend“ 
zu diskutieren, sondern sich auch mit der in diesem Beitrag vorgetragenen Position Flitners auseinanderzusetzen. Gleichzeitig bietet der Text vielfältige Anknüpfungspunkte: Zur Geschichte der Pädagogik (etwa: Reformpädagogik), zur Lernpsychologie (etwa: Motivation und Selbstvertrauen) oder zur Theorie der Schule.

Mit den in dieser Publikation versammelten Texten wird also beabsichtigt, das umzusetzen, was der Titel verspricht: „Einführung in pädagogisches Sehen und Denken“. Im Nachwort der Herausgeber zur überarbeiteten Neuausgabe von 1984 einige Texte wurden ersetzt - heißt es sodann: „Mit diesem Band haben wir den Versuch unternommen, in das Studium der Pädagogik auf andere Art einzuführen als durch ihre Darstellung in einem vereinfachten System. Eine Systematische oder Allgemeine Pädagogik kann eher am Ende als am Anfang des Studiums stehen." (ebd., S. 239)

Es ist wohl nicht untypisch, dass diese Einführung in ein Sehen und Denken in enger Verbindung steht mit einer Professionsorientierung, wie sie sich in diesem Studiengebiet zeigt. Solche Einführungen zielen dann nicht nur auf eine disziplinäre Identität $a b$, oftmals geht es hierbei auch darum, die richtige Einstellung im Sinne einer Disziplin resp. hauptsächlich der einer Profession inhärenten „Core Values"zu gewinnen. Die Texte illustrieren dann Verhaltensweisen, die in gewissem Sinne als vorbildlich gesehen werden können, die aber gleichzeitig in ihrem moralisierenden und bisweilen überhöhten Anspruch nicht unproblematisch sind.

„Fälle“ als Zugänge in die Denk- und Arbeitsweise einer Disziplin haben sich in einigen Disziplinen resp. Studiengängen als didaktisches Prinzip etabliert, Kasuistik oder Fallarbeit (case method, case-based learning) hat in diesen Disziplinen eine große Bedeutung. Gerade in der Rechtswissenschaft hat die Arbeit an Fällen eine lange Tradition, ebenso in der Medizin - wobei der Begriff des „Falles“klarer bestimmt ist als bei der unspezifischen Verwendung beim Beispiel Elternabend. Typischerweise sind solche fallorientierten Formen des Lehrens und Lernens insbesondere in professionsorientierten Studiengängen zu finden, bei denen die angemessene Interpretation von Situationen und Fällen bereits zu einem wesentlichen Teil zur Lösung des Problems beiträgt und gleichzeitig oftmals notwendigerweise Abwägungen getroffen werden müssen. ${ }^{1}$

\footnotetext{
${ }^{1}$ Was beispielsweise die Lehrerinnen- und Lehrerbildung betrifft, so haben sich in den letzten Jahren (und damit seit der „Einführung“von Flitner und Scheuerl) die Dokumentation von Fällen mit Audio- und Videodateien und damit die Möglichkeiten fallbasierten Arbeitens enorm erweitert, gleichzeitig aber auch ihr Anspruch als Teil der Professionalisierung künftiger Lehrpersonen verändert.
} 
Der methodische Zugang über den Fall kombiniert hier ein Sehen und Denken mit einer spezifischen Form der Bearbeitung, wie diese eben in einer Disziplin gepflegt wird. Insofern geht die Fallbearbeitung in den Rechtswissenschaften und in der Medizin auch deutlich über das „Fallverstehen“des Beispiels Elternabend hinaus: Es werden damit gleichzeitig disziplinäre Techniken eingeübt, die im Laufe des Studiums in ihrer Wissensbasis angereichert und in ihrer Umsetzung verfeinert werden.

Um Einübung in eine wissenschaftliche Arbeitsweise und Grundhaltung geht es auch beim Forschenden Lernen, das in den letzten Jahren gerade im Zusammenhang mit der Studieneingangsphase vermehrt diskutiert wird (vgl. zum Beispiel Reinmann et al. 2019). Forschen wird hier zum Modus des Lernens und verbindet dieses Lernen mit den wissenschaftlichen Gepflogenheiten einer Disziplin. Das Forschende Lernen leistet damit - so die Absicht - einen Beitrag zur Wissenschaftssozialisation, indem wissenschaftliche Tätigkeiten eingeübt, disziplinäres Wissen aufgebaut und akademische Grundhaltungen weiterentwickelt werden. ${ }^{2}$ Im deutschen Sprachraum ist dieser Zugang „Forschendes Lernen“ hauptsächlich durch die Schrift der Bundesassistentenkonferenz (Bundesassistentenkonferenz 2009/1970) lanciert worden (vgl. Beitrag Tremp, in diesem Band). Betont wird dort ein Verständnis von Wissenschaft als Vollzug, die Teilnahme an diesem Vollzug und also am Erkenntnisprozess ist damit zentrales Postulat.

Mit dem Bezug auf Forschung verknüpft sich das Forschende Lernen in enger Weise mit Wissenschaftlichkeit. In verwandter Weise findet sich dies auch beim „Genetischen Lernen“, eine Form, welche in der erwähnten Schrift der Bundesassistentenkonferenz zwar dem Forschenden Lernen gegenübergestellt, aber ebenfalls als Zugang zur Wissenschaftssozialisation beschrieben wird: „An sich bekannte Resultate werden ... gleichsam noch einmal in den Prozess zurück verwandelt, der zu ihnen führte; dass Wissenschaft auch hier als Prozess erscheint, problembezogen ist und entsprechende Verhaltensformen fordert" (ebd., S. 24). Genetisches Lernen beabsichtigt also ebenfalls den Aufbau wissenschaftlicher Verhaltensweisen und damit Einführung in ein wissenschaftliches Arbeitsverständnis. Gleichzeitig wird hier die „Genesis“wissenschaftlicher Erkenntnisse der jeweiligen Disziplin nachvollzogen, indem der Student resp. die Studentin

\footnotetext{
${ }^{2}$ Mit Forschendem Lernen in der Studieneingangsphase sind zudem eine Reihe von weiteren Zielsetzungen verbunden, wie dies kürzlich die empirische Analyse von Lübcke und Heudorfer (2019) gezeigt hat.
} 
noch einmal vor das Problem gestellt wird, das den tatsächlichen (historischen) oder einen analogen Ausgangspunkt der betreffenden Forschungsarbeit darstellt, und indem er ferner den Forschungsweg mit den wichtigsten Stationen (Entscheidungspunkten) noch einmal mehr oder weniger frei durchläuft und dabei in ähnlicher Weise tätig ist, wie er es in der originalen Forschungssituation sein müsste (ebd, S. 24).

Studienbücher, Fälle, Forschungshandwerk: Die unterschiedlichen Möglichkeiten eines Einstiegs in die Wissenschafts- und Hochschulwelt haben sich nicht zuletzt darin zu bewähren, inwiefern es gelingt, „Wissenschaft (...) als ein noch nicht ganz aufgelöstes Problem" zu behandeln und damit die Besonderheit dieser Bildungsstufe resp. Bildungseinrichtung erfahrbar zu machen. Dies gilt auch für die Auseinandersetzung mit Klassikern, die ebenfalls zum Zwecke der Wissenschaftssozialisation fungieren können.

\section{$2 \quad$ Klassiker als anregender Ausgangspunkt}

Klassiker-Zusammenstellungen sind oftmals zum Zwecke der Wissenschaftssozialisation geschrieben. In dieser Funktion der Einführung in die Denkweise und Entwicklung einer Disziplin sind sie keine neue Textgattung. Was die Pädagogik betrifft, so gibt es bereits zur Zeit ihrer Entstehung als eigenständige Disziplin um 1800 Hinweise auf lesenswerte Autoren (so zum Beispiel im Werk „Allgemeine Revision des gesamten Schul- und Erziehungswesens"), und Scheidig (in diesem Band) weist in seinem Beitrag beispielsweise auf die nicht unbescheiden angekündigte Sammlung „Pädagogische Klassiker. Auswahl der besten pädagogischen Schriftsteller aller Zeiten und Völker"(1877-1888) von Gustav Adolf Lindner hin.

Klassikersammlungen finden sich bis heute - wenn auch vermehrt mit Spezifikationen und teilweise unter Vermeidung des Begriffs. Eine Auswahl der Titel illustriert dieses Genre:

- Klassiker der Pädagogik

- Klassiker der Pädagogik der frühen Kindheit

- Klassiker der Sozialpädagogik

- Verdrängte Klassiker und Klassikerinnen der Pädagogik

- Klassiker und Außenseiter. Pädagogische Veröffentlichungen des 20. Jahrhunderts 
- Zeitgemäße Klassiker der Pädagogik

- Bildung und Erziehung. Von Klassikern lernen

- Schlüsselwerke der Pädagogik

Es scheint, als hätten einführende Publikationen in (vor allem) sozial- und geisteswissenschaftlichen Disziplinen zugenommen - und damit auch Sammlungen zu Klassikern eines Studienfachs. Zu vermuten ist, dass dies in der Absicht unternommen wurde, die Grundfragen einer Disziplin und deren Geschichte kennenzulernen. In der evolutionstheoretischen Argumentation Tremls erhalten solche Publikationen resp. Studienbücher eine spezifische Bedeutung: Das Curriculum, das sich in solchen einführenden Zusammenstellungen zeigt,

verkörpert hier die kulturelle Evolution; die Schritte der Variation - Selektion - Stabilisierung hat es schon hinter sich. Das kodifizierte Ergebnis dieses evolutionären Prozesses einer Kultur ist in den offiziellen Lehrplänen nachlesbar. Dem individuellen Schüler aber tritt das kulturelle Evolutionsprodukt immer nur als ein äußerer Anspruch gegenüber, als ein Anspruch auf weitere Evolution. Jede weitere Evolution geht durch das Nadelöhr individueller Lernprozesse hindurch und ist, weil diese nur autopoietisch ablaufen können, immer unsicher, unvorhersehbar, unprognostizierbar. In jeder neuen Generation entscheidet sich damit immer wieder aufs Neue, was von den traditionellen Bildungsgütern weiter tradiert wird, ob ein Klassiker ein Klassiker bleibt oder aber zu einem Gegenstand eines bloß historischen Interesses herabsinkt (Treml 1997, S. 136 f.).

Franke-Meyer und Reyer betonen dann auch die curriculare Bedeutung der Klassiker eines Faches, wenn sie in ihrer Einleitung explizit nach dem Beitrag von Klassikern „bei der Sozialisierung des professionellen und disziplinären Nachwuchses und ihrer Einübung in den Korpsgeist eines Faches" (Franke-Meyer und Reyer 2015, S. 8) fragen. Klassiker könnten nur „,durch immer erneute Rezeptions- und Erschließungsarbeit“ ihre Wirkung entfalten. Um aber „Breitenwirkung zu sichern, müssen sie auf allen Ebenen der Sozialisierung des Nachwuchses in Profession und Disziplin präsent sein, das heißt sie müssen curricularen Wahlpflichtstatus haben. Das schließt ein, dass die wichtigsten Schriften der Klassiker gelesen werden, zumindest aber doch in ihrem thematischen Grundgehalt bekannt sein sollten." (Franke-Meyer und Reyer 2015, S. 8) ${ }^{3}$

\footnotetext{
${ }^{3}$ Dabei verweisen die Autorin und der Autor auch darauf, dass dieser Umgang mit Klassikern gerade im Konstitutionsprozess der „Kindheitspädagogik“ als erziehungswissenschaftliche Teildisziplin bedeutsam sei (Franke-Meyer und Reyer 2015, S. 8).
} 
Dass Klassiker eine didaktische Funktion haben, wird in vielen Einleitungen zu entsprechenden Sammelwerken oder Beiträgen betont. Wie wird dies konkret expliziert, welche Überlegungen beziehen sich auf die didaktische Funktion einer Wissenschaftssozialisation? Dies soll anhand ausgewählter Beispiele aus der Disziplin Erziehungswissenschaft illustriert werden. Dabei wird hier davon ausgegangen, dass es Klassiker gibt; für die Zielsetzung dieses Beitrags ist es sekundär, wer denn nun die Klassiker einer Disziplin seien und wie sich diese etablieren konnte. Gefragt wird hier nach der didaktischen Bedeutung im Rahmen der Wissenschaftssozialisation in einem disziplinär strukturierten Hochschulstudium.

Für Winkler „,konstituiert sich der Klassiker im Zusammenhang eines Diskurses mit sozialen Praktiken, der einer doppelten Dialektik unterliegt. Einmal kennzeichnet diesen Zusammenhang die Dialektik von Universalität und Besonderheit; zum anderen aber verbindet er die von Autorität und Diskursivität. Der Klassiker verbindet beide und gewinnt daraus seine Normativität." (Winkler 1994, S. 156) Im Zusammenhang mit der „Disziplinierung der Disziplin“ übernehmen Klassiker bedeutsame Funktionen. So wird mit ihnen beispielsweise die Differenz zwischen Ansprüchen und gegenwärtigen Realisierungen verdeutlicht und damit gegen außen Distanz, gegen innen aber Einschluss angestrebt.

Dollinger diskutiert die Funktion der Klassiker ,für die Einsteiger in ein Fachgebiet" (Dollinger 2012, S. 7) und unterstellt, dass sich die Auseinandersetzung mit ihnen lohne, da sie ein Wissen repräsentieren, das sich nicht leugnen lasse, und Fragen aufwerfen von grundlegender Relevanz (ebd., S. 8). Er erörtert die nicht unproblematische Fokussierung auf Klassiker als Personen (,,personale Mystifizierung“), die ihre Lösungen doch „ohne den Gedankenaustausch mit Personen, die ebenfalls an diesen Problemen arbeiteten, (...) nicht hätten finden können“ (ebd., S. 17). Umgekehrt erleichterten diese Personalisierungen „den didaktischen Zugang zu mitunter komplexen Problemen. Sie ermöglichen Interpunktionen und stellen klar umrissene Bezugspunkte her wie die Lebensspanne einer Person, ihre Interessen, Verschiebungen thematischer Auseinandersetzungen usw." (ebd., S. 17) Sie würden in gewisser Hinsicht als Ganzheit wahrgenommen und konstituieren eine ,pädagogische Zeit“".

Wie sehr Dollinger auch den Anregungsgehalt von Klassikern gewichtet, zeigt sich im abschließenden vierten Teil seiner Klassikersammlung, der mit Foucault, Bourdieu und Luhmann Wissenschaftler „,noch nicht als Klassiker im engeren Sinne“ vorstellt und dies „als Angebote für Klassikerkonstruktionen“ versteht. „Sie vermögen in besonderem Masse zu zeigen, dass die Beschäftigung mit Klassikern alles andere ist als Reflexionsarbeit in konservierender Absicht, sondern mitunter überraschende Einsichten bietet und zu neuen Erkenntnissen zu verhelfen vermag." (ebd., S. 22) 
Für Treml reduzieren und eröffnen Klassiker Komplexität in einem. „Sie reduzieren Komplexität, weil man bei ihnen beginnen (oder enden) kann und damit immer mehr aus- als einschließt; sie eröffnen Komplexität, weil man, von ihnen ausgehend, beliebig weiterschreiten, d. h. weiterdenken kann." (Treml 1997, S. 87) Insofern - darauf macht auch Zierer (2005, S. 270) aufmerksam - zeigen uns Klassiker angesichts unbegrenzter Lektüremöglichkeiten, womit wir beginnen können, ohne damit aufhören zu müssen.

Dabei wendet sich Treml auch gegen die ,,verbreitete Einschätzung“, ,,dass der Gedanke im klassischen Text schon ein für allemal enthalten, wenngleich auch meistens sehr tief verborgen ist und nur wie ein Schatz gehoben werden kann bzw. aufgefunden und entschlüsselt werden muss.“ (Treml 1997, S. 99 f.) Vielmehr würde uns die Klassikerlektüre zum Selbst-Denken stimulieren - ,und bei wirklich brillanter Literatur um so mehr, je öfter wir sie lesen“ (ebd., S. 99).

Damit gleicht die eigene Denkarbeit, die einem durch Klassiker aufgegeben ist, auch derjenigen der Klassiker selber: „Auch Klassiker haben immer nur selbst gedacht, und sie sind vermutlich u. a. gerade deshalb zu Klassikern geworden, weil sie dieses Selbstdenken in bewunderungswürdiger Weise kultiviert haben.“ (Treml 1997, S. 100) Dabei ist allerdings zu berücksichtigen, dass auch die Klassiker nicht bei Null begonnen haben, vielmehr stehen auch ihre Überlegungen in einer Denktradition.

Dabei geht es keinesfalls um das - in Verwendung eines Luhmann-Zitates „,klassische ,Wiederaufwärmen und Immer-wieder-Abnagen der Knochen der Klassiker“", dies sei vielmehr ,ein hermeneutisches Missverständnis“ (ebd., S. 100). Denn das vornehmste Ziel der Klassiker ist vielmehr die Irritation, „Erziehung zum respektlosen Umgang mit den Abenteurern des menschlichen Geistes, die wir ,Klassiker' nennen.“" (ebd., S. 140)

Hellekamps (2017) unterscheidet originellerweise zwischen „Klassikern für Anfänger“ und „Klassikern für Fortgeschrittene“ und betont je spezifische Funktionen. ${ }^{4}$

Für Anfänger haben Klassiker die Bedeutung, dass sich hier ,geeignete Problemformulierungen“ (Hellekamps 2017, S. 6) vorfinden, „um grundlegende Fragen des Fachs zu erläutern“ und „,diese Fragen in ihrem für die Pädagogik paradigmatischen Charakter zu explizieren“ (ebd., S. 6). Für Anfänger ist dabei auch bedeutsam, dass hier - bei Kant, Humboldt oder Schleiermacher - ,ein erzieherisches Problem frei von terminologischem Ballast formuliert" wird.

\footnotetext{
${ }^{4}$ Während das Kapitel „Klassiker für Anfänger“ lediglich eineinhalb Seiten umfasst, wird das Kapitel „Klassiker für Fortgeschrittene“ auf über neun Seiten abgehandelt.
} 
Die Formulierungen sind noch relativ nah an der Alltagssprache; der Text ist nicht mit wissenschaftlicher Terminologie aufgeladen und überfrachtet. Das bedeutet nicht, dass klassische Texte in ihrer Komplexität unmittelbar verständlich wären und keiner hermeneutischen Vergewisserung über ihren jeweiligen historischen Verstehenshorizont bedürften. Aber ein spezifischer wissenschaftstheoretischer Kontext muss nicht vorab bekannt sein (ebd., S. 7).

Und:

Zugleich sind die Begriffe, die pädagogische Klassiker anbieten, oft durch eine deskriptiv-normative Doppelstruktur gekennzeichnet. Denn es geht zumeist nicht nur darum, deskriptive Unterscheidungen $\mathrm{zu}$ treffen, sondern auch spezifische erwünschte Formen anzudeuten, in denen Erziehung erfolgen soll (ebd.).

„Klassiker für Fortgeschrittene“ übernehmen eine andere Funktion. In Anlehnung an Überlegungen zu „Strukturen pädagogischer Handlungstheorie“ (1980) von Friedhelm Brüggen - ihm ist der Beitrag gewidmet - geht Hellekamps davon aus, dass die Trennung von theoretischer und praktischer Vernunft nicht aufgehoben, sondern als „Spezifikum der kognitiven Struktur der Moderne“ (Hellekamps 2017, S. 8) zu berücksichtigen sei. Ins Zentrum gerückt wird sodann der Begriff der Überzeugungen. ${ }^{5}$ Überzeugungen sind eine Form des „Fürwahrhaltens“ und beanspruchen „moralische Gewissheit“ - im Gegensatz zur logischen Gewissheit des Wissens. Überzeugungen sind „Verpflichtungen auf Normen und Werte“ (ebd., S. 11), ihre Rechtfertigung beruft sich auf Instanzen, ,die von vernünftigen Personen für prinzipiell anerkennenswert gehalten werden können“ (ebd., S. 11).

Die Bedeutung von Klassikern liegt nun - im Rahmen dieser Argumentation - darin, dass Klassiker als ,verschriftlichte, kodifizierte und kanonisierte Bestandteile von Überzeugungssystemen“ gelten können. Hier haben sich Überzeugungen in einem Rationalisierungsprozess aus ihrem ursprünglichen pragmatischen Zusammenhang herausgelöst, die grundsätzlichen Überlegungen wurden in ein weiter entwickeltes „Überzeugungsgefüge“ (ebd., S. 15) integriert - als Quelle für Sinnorientierung. Klassikerlektüren in einem Studium hätten dann die Bedeutung, dass durch diese ,die Lernenden eine Sprache finden können, die ihnen ihre eigenen Anliegen erst zu Bewusstsein bringt“ (ebd., S. 17). „Klassiker können den Lernenden helfen, ihre Überzeugungen, also ihr begründetes

\footnotetext{
5Überzeugungen sind nicht gleichzusetzen mit „,beliefs“oder „subjektiven Theorien“, die stets als defizitäre Formen epistemischen Wissens in den Blick geraten und damit notwendigerweise bearbeitet werden müssen.
} 
Fürwahrhalten, in Hinsicht auf die pluralen Sinnangebote unserer modernen Welt zu formen, zu relativieren, zu differenzieren“ (ebd., S. 18). Klassiker würden also, so das Fazit von Hellekamps, ,auch für professionelle pädagogische Akteure eine Sprache bereithalten, die dabei hilft, den Kern unserer Probleme mit der Moderne herauszuschälen und unsere Fragestellungen zu pointieren. Klassiker stehen mit ihren problematisierenden Formulierungen, die sich auch für Erziehungswissenschaftler und Erziehungswissenschaftlerinnen bereithalten, am Beginn eines Reflexionsprozesses. Aber in der Gegenwart muss der Erziehungswissenschaftler seine eigene Antwort geben." (ebd., S. 18)

Insgesamt, so darf resümierend festgehalten werden, wird Klassikern in diesen ausgewählten (und exemplarisch zu verstehenden) Beiträgen also eine bedeutsame Funktion zugeschrieben, was die Wissenschaftssozialisation einerseits und damit verbunden gleichzeitig die Reproduktion der Disziplin und ihres gedanklichen Kerns andererseits betrifft.

Betont wird Diskursivität. Klassiker sind kein heiliger Textkorpus, sondern lebendige Anregung und geeignet zur Differenzierung der eigenen Konzepte und Vorstellungen. Und die Auseinandersetzung mit Klassikern wird als Einladung verstanden, sich an disziplinären Diskussionen und also Weiterentwicklungen zu beteiligen. Klassiker haben hochschuldidaktisch also dann ihre Bedeutung, wenn sie Auseinandersetzung beabsichtigen. Dabei kann sich kein Klassiker seines Status sicher sein, dieser Status steht stets grundsätzlich zur Debatte. Nur dies wird dem Anspruch und der Eigentümlichkeit einer Hochschuldidaktik gerecht: Während eine Didaktik der Schule insbesondere bestrebt ist, zum Zwecke des Lehrens und Lernens Komplexität der Sache zu reduzieren, ist Hochschuldidaktik dadurch charakterisiert, dass hier Komplexität expliziert wird und damit zum vertieften Verständnis der Sache beiträgt - oder sogar zu einem anderen Verständnis beiträgt, was sich didaktisch als Dialektik von Kanon und Wissenskritik beschreiben lässt (Eugster und Tremp 2018).

Bei dieser wissenschaftssozialisatorischen Bedeutung von Klassikern ist man geneigt, der Einschätzung Tremls zuzustimmen: „In Anbetracht ihrer pädagogischen Relevanz und ihrer schwierigen und sperrigen Zugänglichkeit ist es erstaunlich, dass die Pädagogik ... keine ausgearbeitete Didaktik klassischer Texte besitzt.“ (Treml 1997, S. 154) $)^{6}$ Denn tatsächlich wird auch immer wieder

${ }^{6}$ Treml (1997) unterscheidet zwei Extreme des Umgangs mit Klassikern: Zum einen eine Didaktik des Ganz-Verstehens, die also versucht, etwas vollständig zu verstehen, bevor nächste Teile angepackt werden. Zum anderen eine Didaktik des antizipierenden Lernens, welche von einer „Zumutung des Nichtverstehens“ ausgeht und Lernen als Herausforderung und Abenteuer versteht. 
auf die Gefahr von Klassikern hingewiesen, sollten diese nicht als anregender Ausgangspunkt des Denkens genutzt werden, sondern im Status einer kanonisierten Heiligkeit präsentiert und diskutiert und damit vor allem ihre identitätsstiftende Tradition ins Zentrum gerückt werden. Klassiker würden nämlich „dazu verführen, mystifiziert und pantheonisiert zu werden, blind zu machen, unproduktiv und unkritisch zu rezipieren, Epigonen zu erzwingen, andere Auffassungen zu diskriminieren"(Zierer 2009, S. 68). Und es wird betont, dass bei dieser didaktischen Funktion der Klassiker die Auswahl von großer Bedeutung ist, denn: „Irrtümer können aufgrund der Macht, die man dem Klassiker zuschreibt, fatale Folgen haben. Wer sich am Falschen orientiert, generiert möglicherweise fehlgeleitete und irrige Perspektiven und stattet sie auch noch mit dem Anspruch ahistorischer Wahrheit aus." (Dollinger 2012, S. 9) ${ }^{7}$

\section{$3 \quad$ Einstiege in die Hochschuldidaktik}

Wenn die Auseinandersetzung mit Klassikern einen Beitrag leisten kann zur disziplinären Sozialisation und Weiterentwicklung, so soll hier nun abschließend geprüft werden, wie sich diese Überlegungen auf die Hochschuldidaktik beziehen lassen. Allerdings muss dabei berücksichtigt werden, dass sich diese im deutschen Sprachraum bisher nicht als Disziplin hat etablieren können. Zwar kennt sie einige Strukturmerkmale von Disziplinen (Fachgesellschaft, Publikationsformate, Tagungen etc.), aber insbesondere kennt sie - mit wenigen Ausnahmen - keine grundständigen Studiengänge, wie dies etwa in der „Bindestrich-Disziplin“ Erwachsenenbildung der Fall ist. In der Hochschuldidaktik - so haben es Fleischmann et al. beschrieben - hätten wir es mit einer „Patchworkprofessionalisierung“" (Fleischmann et al. 2016) zu tun. Damit ist auch ausgedrückt, dass Hochschuldidaktikerinnen und Hochschuldidaktiker nicht nur aus unterschiedlichen Disziplinen in dieses Arbeitsfeld hineinkommen, sondern auch, dass kaum ein geteiltes Selbstverständnis entwickelt ist.

Tatsächlich würden „Klassiker der Hochschuldidaktik“ - würde es denn solche geben - kein Anwendungsfeld in Bachelor-Studiengängen finden und damit

\footnotetext{
${ }^{7}$ Zwar zeigt sich in der Disziplin Pädagogik ein Set an Autorinnen und Autoren oder Texten, die unbestritten zu sein scheinen, ohne aber die Grenzen dieses Kreises streng bestimmen zu können. Entsprechend gibt es auch immer wieder Ergänzungen zur traditionellen Klassikerliteratur. Etwa, wenn an „,verdrängte Klassiker“ (Kluge und Borst 2013) erinnert wird oder an „Außenseiter“ (Horn 2003), die eben zu wenig berücksichtigt und beachtet würden.
} 
die „klassische“ wissenschaftssozialisatorische Funktion übernehmen können. Die Schwierigkeiten der Hochschuldidaktik mit der Klassikerfrage dürften aber auch durch ihr Fremdeln mit der Geschichte sogar vergrößert sein. Hochschuldidaktik kann zwar auf eine viele Jahrhunderte zurückreichende Lehrpraxis rekurrieren, stets auch begleitet durch theoretische Erörterungen dieser Praxis, gleichwohl ist diese Geschichte innerhalb der Hochschuldidaktik wenig bekannt. $^{8}$

Klassikernennungen, so hat Dollinger dies beschrieben, seien auch stets ,strategische Markierungen“ (Dollinger 2012, S. 18), hier wird ein Feld abgesteckt und gleichzeitig auf Zustimmung im wissenschaftlichen Kollegenkreis gesetzt. Damit wird auch Abgrenzung gepflegt: Wer gehört dazu, wer kennt die Namen und wer eben nicht.

Grundsätzlich stellt sich die Frage, wer denn überhaupt Klassiker braucht: Sind diese für Dozierende bedeutsam? Und für Hochschuldidaktikerinnen und Hochschuldidaktiker? Oder für am Fach interessierte Personen, die eine erste Orientierung gewinnen wollen?

Es ist zu vermuten, dass Dozierende kaum Bedarf an „Klassikern der Hochschuldidaktik" haben zum Zwecke der Wissenschaftssozialisation. Ihre wissenschaftliche Orientierung erfolgt in ihrer Disziplin, Lehre ist hier eine bestimmte „Verwendungsform des Wissens“ (Tremp et al. 2014). Didaktik als Wissenschaft des Lehrens muss für sie handlungsleitend und aufbereitet zur Verfügung stehen. Klassiker dienten wohl vor allem dafür, sich im Rahmen von hochschuldidaktischen Qualifizierungsverfahren hochschuldidaktischer Methoden in ihrer Tradition zu versichern. ${ }^{9}$

Tatsächlich lässt sich auch feststellen, dass sich die meisten Einführungen in die Hochschuldidaktik als Einführungen in die Vielfalt methodischer Gestaltung von Lehre verstehen - inzwischen verstärkt angereichert mit empirischen

\footnotetext{
${ }^{8}$ Oder einfach kein Thema, weil sie keine Disziplin ist, sondern ein Arbeitsfeld, das weder Zeit noch Auftrag für eine historiografische Betrachtung einer ihrer Praxisfelder - der Hochschullehre - hat. Gemeint ist damit der durchaus merkwürdige Befund: Die Genese der Hochschuldidaktik lieferte kaum Gründe und Gelegenheit für eine Auseinandersetzung mit der Genese der Hochschullehre.

${ }^{9}$ Dozierende seien, so Kandlbinder (2013), höchst zurückhaltend gegenüber hochschuldidaktischer Literatur. Allerdings zeigten sich hierbei auch benennbare Herausforderungen. Dazu gehöre, dass es nicht einfach sei, bedeutsamere von weniger bedeutsamen, zentrale von peripheren Beiträgen zu unterscheiden. Entsprechend verbindet Kandlbinder mit seinen Beiträgen zu den ,signature concepts“ die Absicht, hier Orientierung für Hochschullehrer zu bieten.
} 
Evidenzen. Es sind Handlungsorientierungen in praktischer Absicht, Klassiker werden ebenso wenig zum Thema wie die Geschichte des Fachs resp. der Hochschullehre und ihrer diskursiven Begleitung.

Das lässt sich an einführenden Werken leicht illustrieren. Diese strukturieren sich oftmals anhand der zentralen Tätigkeitsfelder von Dozierenden und thematisieren also Aufgaben der Planung von Lehrveranstaltungen, die konkrete Realisierung in unterschiedlichen Veranstaltungsformaten oder Prüfungen und Lehrevaluation. Vielfach machen bereits die Untertitel dieser Publikationen auf Zielsetzung und Fokus der Praxisanregung aufmerksam. ${ }^{10}$ Solche praktischen Anleitungen sind typisch für die hochschuldidaktischen Publikationen, die sich an Dozierende richten. Sie sind Handreichungen, keine Einladungen zur Beteiligung an der hochschuldidaktischen Diskussion. ${ }^{11}$

Bisweilen lässt sich nicht immer deutlich unterscheiden, ob sich solche hochschuldidaktischen Publikationen an Dozierende oder an Hochschuldidaktikerinnen oder Hochschuldidaktiker richten - oder an beide gleichermaßen. Dies mag nicht zuletzt mit der erwähnten „Patchworkprofessionalisierung“ zu tun haben resp. mit dem ungeklärten Disziplincharakter der Hochschuldidaktik.

Damit sind wir zurückverwiesen auf die wissenschaftliche Sozialisation von Hochschuldidaktikerinnen und Hochschuldidaktikern. Vor wenigen Jahren hat Gabi Reinmann einen Vorschlag vorgelegt für „Wissenschaftliche Lektüre zum Einstieg in die Hochschuldidaktik“ (Reinmann 2016). Dieser Vorschlag steht insbesondere in einem engen Zusammenhang mit einem Studienangebot am Zentrum für Universitäres Lehren und Lernen HUL an der Universität Hamburg, das die Teilnehmerinnen und Teilnehmer zu einer vertieften Auseinandersetzung mit der Disziplin Hochschuldidaktik anregen will. Oder wie es Gabi Reinmann zusammenfasst: „In jedem Fall aber sollte, so meine Hoffnung, die Lektüre die Teilnehmerinnen zu Beginn des Masterstudiums darin unterstützen, das wissenschaftliche wie auch praktische Potenzial der Hochschuldidaktik zu erkennen und einschätzen zu lernen“" (ebd., S. 1).

\footnotetext{
${ }^{10}$ So zum Beispiel bei der (meines Erachtens hervorragenden!) Einführung von Götz Fabry (2008) in die Medizindidaktik. Die Einführung startet mit einem Problemaufriss (,Welche Ausbildung braucht ein Arzt?"), präsentiert anschließend einige lernpsychologisch-didaktische Überlegungen und thematisiert dann insbesondere die verschiedenen Tätigkeitsfelder von Hochschuldozierenden.

${ }^{11}$ Interessant könnte hierbei auch eine Prüfung von Literaturlisten sein, welche in hochschuldidaktischen Weiterbildungsveranstaltungen verwendet werden - gerade auch im Zusammenhang mit hochschuldidaktischen Qualifizierungsprogrammen.
} 
Vorgeschlagen sind hier sechs Texte, welche „bewusst aus verschiedenen Zeiten stammen und unterschiedliche Akzente setzen, aber einen ähnlichen Abstraktionsgrad und einen gemeinsamen Zweck haben: Es geht in allen Texten nicht um die Praxis der Hochschullehre, sondern darum, wie man sich wissenschaftlich mit eben dieser auseinandersetzen kann." (ebd., S. 1) Damit sind zwar keine „Klassiker“ genannt, aber immerhin ist hier eine Zielsetzung formuliert, die „Wissenschaftssozialisation“ beabsichtigt und also Hochschuldidaktik als Wissenschaft zum Thema macht und eine „heimische“ Diskussion beabsichtigt.

Diese theoretische Auseinandersetzung mit der Disziplin resp. dem Fach Hochschuldidaktik setzt ein bestimmtes (Vor-)Verständnis von Hochschuldidaktik voraus, damit der Kern des Gegenstandes und seine Grenzen bestimmbar werden. Hochschuldidaktische Klassiker finden sich wohl erst, wenn auch die Hochschuldidaktik ihre heimischen Fragen klärt. Und sind diese geklärt, so können Fragen von der eigenen Disziplin her formuliert werden - selbst die Lektüre unterschiedlichster Autorinnen und Autoren wird dann zur Anregung. So hat bereits der Philanthrop Johann Stuve in seinem Beitrag „Allgemeinste Grundsätze der Erziehung“ (1785; publiziert im Werk „Allgemeine Revision des gesamten Schul- und Erziehungswesens“) die Bedeutung der ,würdigsten Denkmäler der Geschichte der Menschheit" betont und dem Pädagogen die Lektüre der Schriften von „Homer, Horaz, Cervantes, Shakespeare, Milton, Pope, Fielding, Klopstock, Lessing, Richardson, Wieland, Stern“ empfohlen und ergänzt „,wenn er kann, so studiere er auch die Rubens und Raphaels in ihren Werken“"(Allgemeine Revision, S. 244).

\section{Literatur}

Allgemeine Revision des gesammten Schul- und Erziehungswesens. 1785-1792. Von einer Gesellschaft praktischer Erzieher; herausgegeben von Joachim Heinrich Campe. 16 Bände, Band 1. Hamburg: C.E. Bohn (https://goobiweb.bbf.dipf.de/viewer/ toc/023270969/0/)

Bundesassistentenkonferenz. (2009/1970). Forschendes Lernen - Wissenschaftliches Prüfen. Ergebnisse der Arbeit des Ausschusses für Hochschuldidaktik. Bielefeld: Universitäts VerlagWebler.

Dollinger, B. (2012). Klassiker der Pädagogik. Einleitende Anmerkungen zu einer eigentümlichen Spezies. In B. Dollinger (Hrsg.), Klassiker der Pädagogik (S. 7-24). Wiesbaden: Springer Fachmedien.

Eugster, B., \& Tremp, P. (2018). Lehre als Zugang zum Fach - Plädoyer für eine didaktische Wissenschaftssozialisation. In M. Weil (Hrsg.), Zukunftslabor Lehrentwicklung. Perspektiven auf Hochschuldidaktik und darüber hinaus (S. 75-93). Waxmann: Münster. 
Fabry, G. (2008). Medizindidaktik. Ein Handbuch für die Praxis. Bern: Huber.

Fleischmann, A., Heiner, M. \& Wiemer, M. (2016). Patchworkprofessionalisierung: Der Einstieg in die Hochschuldidaktik. In M. Heiner, B. Baumert, S. Dany, T. Haertel, M. Quellmelz \& C. Terkowsky (Hrsg.), Was ist „Gute Lehre“? Perspektiven der Hochschuldidaktik (= Blickpunkt Hochschuldidaktik; 129), (S. 97-109). Bielefeld, Bertelsmann.

Flitner, A., \& Scheuerl, H. (Hrsg.). (1984). Einführung in pädagogisches Sehen und Denken. Piper: München.

Franke-Meyer, D., \& Reyer, J. (Hrsg.). (2015). Klassiker der Pädagogik der frühen Kindheit. Ideengeber und Vorläufer des Kindergartens. Juventa: Weinheim.

Hellekamps, S. (2017). Propädeutik, Problematisierung und Überzeugungen. Zur Bedeutung von Klassikern in der Pädagogik. Für Friedhelm Brüggen zum 65. Geburtstag. Zeitschrift für Erziehungswissenschaft, 20, 5-20.

Horn, K.-P. (Hrsg.). (2003). Klassiker und Aussenseiter: Pädagogische Veröffentlichungen des 20. Jahrhunderts. Baltmannsweiler: Schneider.

Humboldt, W. (1810/1964). Über die innere und äußere Organisation der höheren wissenschaftlichen Anstalten in Berlin. In von A. Flitner \& F. Giehl (Hrsg.), Werke in fünf Bänden, Band IV, Schriften zur Politik und zum Bildungswesen(S. 255-266). Darmstadt: Wissenschaftliche Buchgesellschaft.

Kandlbinder, P. (2013). Signature concepts of key researchers in higher education teaching and learning. Teaching in Higher Education, 18(1), 1-12.

Kluge, S., \& Borst, E. (Hrsg.). (2013). Verdrängte Klassiker und Klassikerinnen der Pädagogik. Baltmannsweiler: Schneider-Verl. Hohengehren.

Lübcke, E., \& Heudorfer, A. (2019). Die Ziele forschenden Lernens Eine empirsiche Analyse im Rahmen der QPL-Begleitforschung. In G. Reinmann, E. Lübcke, \& A. Heudorfer (Hrsg.), Forschendes Lernen in der Studieneingangsphase Empirische Befunde, Fallbeispiele und individuelle Perspektiven (S. 17-58). Springer: Wiesbaden.

Reinmann, G. (2016). Wissenschaftliche Lektüre zum Einstieg in die Hochschuldidaktik. Impact Free - Journal für freie Bildungswissenschaftler, 5, 7 Seiten. https://gabi-reinmann.de/wp-content/uploads/2016/05/Impact-Free-5.pdf.

Reinmann, G., Lübcke, E., \& Heudorfer, A. (Hrsg.). (2019). Forschendes Lernen in der Studieneingangsphase. Empirische Befunde, Fallbeispiele und individuelle Perspektiven. Wiesbaden: Springer.

Treml, A. K. (1997). Klassiker. Die Evolution einflussreicher Semantik. Theorie. Sankt Augustin: Academia.

Tremp, P., Stäuble, E., \& Suter, A. (2014). Personal und Personalentwicklung in (berufsorientierten) Hochschulen - Ein Diskussionsbeitrag. fh-ch, 3, 9-11.

Winkler, M. (1994). Ein geradezu klassischer Fall. Zur Traditionsstiftung in der Pädagogik durch Klassiker. In K. P. Horn \& L. Wigger (Hrsg.), Systematiken und Klassifikationen in der Erziehungswissenschaft (S. 141-168). Weinheim: Deutscher Studien Verlag.

Zierer, K. (2005). Das Verschwinden eines Klassikers. Vierteljahresschrift für wissenschaftliche Pädagogik, 81(3), 258-281.

Zierer, K. (2009). Vom Nutzen und Nachteil der Klassiker für die Pädagogik. Pädagogische Rundschau, 63(1), 67-73. 
Prof. Dr. Peter Tremp Leiter Zentrum für Hochschuldidaktik an der Pädagogischen Hochschule Luzern. Von 2011-2017 Leiter Abteilung Forschung und Entwicklung der Pädagogischen Hochschule Zürich, von 2004-2011 Leiter der Hochschuldidaktik der Universität Zürich.

Arbeitsschwerpunkte: Hochschuldidaktik, Akademische Bildung, Forschungsorientierung in Studium und Lehre. 